\title{
Implementing an undergraduate research apprenticeship with the Macintosh computer
}

\author{
JEAN L. HATTEN and ANNA D. HATTEN \\ Averett College, Danville, Virginia
}

\begin{abstract}
We discuss the use of Macintosh computers, experiment-building software, and the Excel worksheet to support an undergraduate research apprenticeship. The system described is easy to learn and use, and handles data electronically, from initial data collection to the final presentation of reports.
\end{abstract}

One way to enhance research in psychology is to use computers to provide for the direct involvement of undergraduates in the process. Undergraduate research participation increases the number of researchers, and ensures that undergraduates are actively involved in learning about psychological principles, research methods, and modern data-management techniques.

Our program is based on an apprenticeship model. Students complete a two- or three-semester sequence of research training that begins with an introductory lab that focuses on principles of learning, basic data acquisition techniques, and presentation of results. This is followed by an intermediate lab that requires each student to design and conduct an original experiment, and to present the results at an in-house poster session. Finally, students may elect to participate in the senior thesis program; these projects are usually extensions of work begun in the intermediate lab.

Because undergraduates must integrate their research efforts with the demands made by routine course requirements, one goal of our program is to make the productivity gains associated with modern software tools accessible to undergraduates, many of whom have little or no background in computer science. Our goal was to support the apprenticeship program with a system that was easy to learn, easy to use, and would handle data electronically from initial data collection through the final preparation of graphs and reports, all without requiring data entry from the keyboard.

\section{IMPLEMENTATION}

\section{The Microprocessor}

After a thorough comparison of the IBM and Macintosh microcomputers, the Macintosh appeared to be superior as a beginner's machine. The Macintosh user interface is highly intuitive; learning one software package often allows students to use a new package without consulting

This project was funded by the NSF CSIP program, Grant CSI8650705. Reprints may be obtained from Jean Hatten, Department of Psychology, Averett College, Danville, VA 24541. the manual. In addition, very powerful software is available for the Macintosh that enables students easily to analyze and present data graphically. Furthermore, cost comparisons at the time of purchase indicated that the Macintosh system and a comparably equipped IBM system did not differ appreciably in price. For these reasons, we chose the Macintosh for the microcomputer component of our system.

\section{Experiment Control System Requirements}

After deciding to equip the undergraduate laboratory with Macintosh Plus computers, we looked for an experiment-control system with the following characteristics. First, the system had to control multiple stations with a single computer, allowing for efficient use of our machines. Second, undergraduates needed, whenever possible, to generate their own experiment-control code without programming. This feature was desired because it requires students to develop a clear understanding of experimental procedures. It also frees faculty from many programming chores, so that available time may be spent discussing issues addressed by the students' experiments. Third, the control system needed to facilitate the collection and analysis of relatively detailed data, so that experiments were not avoided due to an inability to collect required measures, and additional measures could be calculated if needed. Finally, due to the limited resources of a small-college environment, the hardware needed to be of the "off-the-shelf" variety.

For effective event control and data collection, the Macintosh Plus requires an intelligent interface with an on-board microprocessor. The available intelligent interfaces for the Macintosh Plus include Reed College's BenchTop, MacLaboratory (Chute, Gaeman, \& Ziegler, 1987), the Walter/Palya networked controller (Cooper, Garcia, \& Gibbon, 1988; Weisman \& Palya, 1988), and International Data Acquisition and Control's IDAC 1000 and IDAC 2000. Both the Walter/Palya board and the IDAC 2000 allow a single Macintosh to control multiple stations, and the cost per station of the IDAC 2000 is roughly the same as a completely assembled Walter/Palya board. The IDAC controllers, however, have the additional benefit of supporting Macontrol software. Macon- 


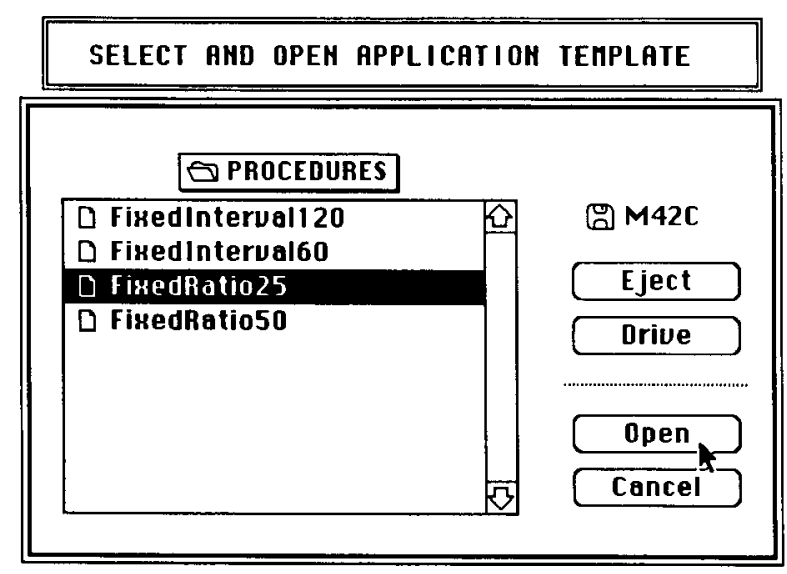

Figure 1. The standard Macintosh "file-open dialog box" being used to open the template of a fixed-ratio 25 schedule in Macontrol. trol permits student-originated experiment control, data collection, and subsequent data analysis, including graphing of data, using a single additional application, the Excel worksheet. For this reason, we decided to purchase the IDAC 2000 unit.

\section{Characteristics of the IDAC 2000}

The IDAC 2000 can address up to 64 optically isolated input/output channels. The Macintosh controls events and acquires data via a high-level command set, resident in the IDAC's read-only memory. The commands allow reading from or writing to individual channels, groups of 8 channels, or groups of 16 channels. Furthermore, the commands allow the user to activate up to 16 on-board counters that can subsequently be read, cleared, or both. In addition, the user may specify a criterion for each of these counters that when met, will ballistically operate a

\begin{tabular}{|c|c|c|c|c|c|c|c|c|c|c|c|c|c|c|}
\hline & $A$ & $\mathrm{~B}$ & $C$ & D & $E$ & $F$ & $T$ & $\mathrm{~J}$ & K & R & 5 & U & W & $\bar{X}$ \\
\hline 1 & MACONTRO & & & & - APPLTC & CATION & TEMPD & ATE & & & & & & \\
\hline 2 & & $S$ & & & & & & & $\#$ & & & & & \\
\hline 3 & & $E$ & $1 / 0$ & on/off/pulse & Output & Channel & & Pulse & of & Freq & & & Timer & Branch \\
\hline 4 & DEVICE\# & $\mathrm{Q}$ & Counter & enable/disable & Address & $(0-7)$ & Ratio & Width & Samp & Count & Crit. & $=$ & (min) & 10: \\
\hline 5 & HLITE\#16 & & 0 & & & & & & & & & 2 & & \\
\hline 6 & KEYLITE\#40 & & 0 & 1 & & & & & & & & 3 & & \\
\hline 7 & KEY\#04 & & $C$ & 7 & 7 & 4 & 5 & 250 & C & . & 40 & 4 & & \\
\hline 8 & KEYLITE\#40 & & 0 & 0 & & & & & & & & 5 & & \\
\hline 9 & HLITE $\# 16$ & & 0 & 0 & & & & & & & & 5 & & \\
\hline
\end{tabular}

Figure 2. A Macontrol application template specifying a fixed-ratio 5 schedule of reinforcement for one experimental chamber.

\begin{tabular}{|c|c|c|c|c|c|c|c|c|c|c|c|c|c|c|}
\hline & $\pi$ & $B$ & $c$ & D & $E$ & $F$ & $T$ & $J$ & K & R & 5 & U & $W$ & $\mathbf{X}$ \\
\hline$T$ & MACONIROL & & & & - APPLIC & CATION & TEMPL & ATE & & & & & & \\
\hline 2 & & $\mathrm{~S}$ & & & & & & & \# & & & & & \\
\hline 3 & & $E$ & $1 / 0$ & on/oft/pulse & Output & Channel & & Pulse & of & Freq & & & Timer & Branch \\
\hline 4 & DEVICE\# & 0 & Counter & enable/disable & Address & $(0-7)$ & Ratio & Width & Samp & Count & Crit. & $=$ & $(\min )$ & 10: \\
\hline 5 & HLITE\#16 & & 0 & 1 & & & & & & & & 2 & & \\
\hline 6 & HLITE\#17] & & 0 & 1 & & & & & & & & 2 & & \\
\hline 7 & HLITE\#18 & & 0 & 1 & & & & & & & & 2 & & \\
\hline 8 & HLITE $\# 19$ & & 0 & 1 & & & & & & & & 2 & & \\
\hline 9 & KEYLITE\#40 & & 0 & 1 & & & & & & & & 3 & & \\
\hline 10 & KEYLITE\#41 & & 0 & 1 & & & & & & & & 3 & & \\
\hline 11 & KEYLITE\#42 & & 0 & 1 & & & & & & & & 3 & & \\
\hline 12 & KEYLITE\#43 & & 0 & 1 & & & & & & & & 3 & & \\
\hline 13 & KEY\#04 & & C & 7 & 7 & 4 & 50 & 250 & C & * & 40 & 4 & & \\
\hline 14 & KEY\#05 & & $\mathrm{C}$ & 7 & 7 & 5 & 50 & 250 & $\mathrm{C}$ & * & 40 & 4 & & \\
\hline 15 & KEY\#06 & & C & 7 & 7 & 6 & 50 & 250 & C & 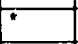 & 40 & 4 & & \\
\hline 16 & KEY\#07 & & C & 7 & 7 & 7 & 50 & 250 & $\mathrm{C}$ & * & 40 & 4 & & \\
\hline 17 & KEYLITE\#40 & & 10 & 0 & & & & & & & & 5 & & \\
\hline 18 & KEYLITE\#41 & & 0 & 0 & & & & & & & & 5 & & \\
\hline 19 & KEYLITE\#42 & & 0 & 0 & & & & & & & & 5 & & \\
\hline 20 & KEYLITE\#43 & & 0 & 0 & & & & & & & & 5 & & \\
\hline 21 & HLITE\#16 & & 0 & 0 & & & & & & & & 5 & & \\
\hline 22 & HLITE\#17 & & 0 & 0 & & & & & & & & 5 & & \\
\hline $2 \sqrt{3}$ & HLITE $\# 18$ & & 0 & 0 & & & & & & & & 5 & & \\
\hline 24 & HLITE\#19 & & 0 & 0 & & & & & & & & 5 & & \\
\hline
\end{tabular}

Figure 3. A Macontrol application template specifying a fixed-ratio 50 schedule of reinforcement for four experimental chambers. 


\begin{tabular}{|c|c|c|c|r|r|r|}
\hline & A & B & C & D & H & T \\
\hline 5 & PROCESS & & & & & \\
\hline 6 & & & & & & \\
\hline 7 & CH\# & SEQ & SEQ & MEAN/ & TIME OF FIRST & END \\
\hline 8 & & NUM & TYPE & PULSES & DIG PULSE(TICS) & TIME(TICS) \\
\hline 9 & $0-16$ & 1 & DO & & & 151106 \\
\hline 10 & $0-40$ & 2 & DO & & & 151119 \\
\hline 11 & $0-4$ & 3 & DE & 50 & 151834 & 152367 \\
\hline 12 & & & & 50 & 152367 & 153117 \\
\hline 13 & & & & 50 & 153130 & 153881 \\
\hline 14 & & & & 50 & 153894 & 154645 \\
\hline 15 & & & & 50 & 154658 & 155526 \\
\hline 16 & & & & 50 & 155526 & 156290 \\
\hline 17 & & & & 50 & 156303 & 157094 \\
\hline 18 & & & & 50 & 157467 & 157947 \\
\hline 19 & & & & 50 & 157960 & 158790 \\
\hline 20 & & & & 50 & 158803 & 159669 \\
\hline 21 & & & & 50 & 159682 & 160459 \\
\hline
\end{tabular}

Figure 4. A segment of the Macontrol data report generated by the fixed-ratio 50 template shown in Figure 3. This figure shows the data obtained from only one of the chambers.

specified output device, such as a pellet dispenser. The high-speed counter option allows sufficiently rapid control of multiple stations by ensuring that responses are sensed in a timely fashion and that there is no unacceptable delay between the target response and the consequence. The Macontrol software takes advantage of these features and, when it is necessary to write custom software for relatively complex procedures, the interface command set is available for applications written in any suitable language.

\section{Features of Macontrol}

To operate Macontrol with existing experiment-control templates, the user chooses a procedure via the standard Macintosh "file-open dialog"; Figure 1 shows that a procedure named FixedRatio 25 has been selected and the user is about to open the file. When the file is opened,
Macontrol prepares a compiled ZBasic program, pauses to request the name of the data volume, and executes the control program.

Creating new program-control templates is simple in Macontrol. For example, to generate a fixed-ratio 5 (FR 5) schedule for a single station, the student would open a special Excel template and then enter the appropriate parameters one row at a time.

Figure 2 shows a template for an FR 5 program. In the figure, the first sequence addresses Houselight 16 (HLITE\#16) as an output, as indicated by the letter $O$ in column $\mathrm{C}$, and turns it on, as indicated by the number 1 in column D. In column $U$, the program is instructed to branch to Sequence 2 .

In the second sequence, Keylight 40 (KEYLITE\#40) is lit. Sequence 3 addresses Pigeon Key 4 (KEY\#04), and is the heart of the fixed-ratio program. Rather than a sim-

\begin{tabular}{|c|c|c|c|c|c|c|c|c|c|c|c|c|c|c|}
\hline & $\bar{A}$ & B & C & D & $E$ & $F$ & T & $\mathrm{J}$ & K & $\overline{7}$ & 5 & U & W & $\mathbf{X}$ \\
\hline 1 & MACONIRO & & & & - APPLT & AIION & TEMPL & ATE & & & & & & \\
\hline 2 & & $\mathrm{~S}$ & & & & & & & $\#$ & & & & & \\
\hline 3 & & $E$ & $1 / 0$ & on/oft/pulse & Output & Channel & & Pulse & of & Freq & & & Timer & Branch \\
\hline$\overline{4}$ & DEVICE\# & Q & Counter & enable/disable & Address & $(0-7)$ & Ratio & Width & Samp & Count & Crit. & $=$ & $(\min )$ & to: \\
\hline 5 & HLITE\#16 & & 0 & 1 & & & & & & & & 2 & & \\
\hline 6 & KEYLITE \#40 & 2 & 0 & 1 & & & & & & & & 3 & & \\
\hline 7 & $\overline{K E Y} \# 04$ & 3 & $\mathrm{C}$ & 5 & & & & & C & " & & & 0.1 & 4 \\
\hline 8 & KEY\#04 & 4 & $\mathrm{C}$ & 5 & & & & & C & + & & & 0.1 & 5 \\
\hline 9 & KEY\#04 & 5 & C & 5 & & & & & C & $\cdot$ & & & 0.1 & 6 \\
\hline 10 & KEY\#04 & 6 & C & 5 & & & & & C & - & & & 0.1 & 7 \\
\hline 11 & KEY\#04 & 7 & C & 5 & & & & & $\mathrm{C}$ & * & & & 0.1 & 8 \\
\hline 12 & KEY\#04 & 8 & C & 5 & & & & & C & * & & & 0.1 & 9 \\
\hline 13 & KEY\#04 & 9 & C & 5 & & & & & C & 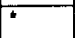 & & & 0.1 & 10 \\
\hline 14 & KEY\#04 & 10 & C & 5 & & & & & C & + & & & 0.1 & 11 \\
\hline 15 & KEY\#04 & 11 & $\mathrm{C}$ & 5 & & & & & C & 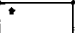 & & & 0.1 & 12 \\
\hline 16 & KEY\#04 & 12 & C & 5 & & & & & C & - & & & 0.1 & 13 \\
\hline 17 & KEY\#04 & 13 & $C$ & 7 & $\overline{7}$ & 4 & . & & $\mathrm{C}$ & * & $\overline{1}$ & $\overline{14}$ & & \\
\hline 18 & KEY\#04 & 14 & & & & & & & C & $\cdot$ & & & 0.07 & 15 \\
\hline 19 & FEEDER\#60 & 15 & 0 & 0 & & & & & & & & 3 & & \\
\hline
\end{tabular}

Figure 5. A Macontrol application template specifying a fixed-interval 60-sec schedule of reinforcement. This template is specifically designed to count responses in each tenth of the interval. 


\begin{tabular}{|c|c|c|c|c|c|c|c|c|c|}
\hline & $A$ & B & C & D & $\mathrm{H}$ & $T$ & $\mathbf{J}$ & $\mathbf{K}$ & L \\
\hline 4 & PROCESS \# & & & & & & & & \\
\hline 5 & $\mathrm{CH} \#$ & SEQ & SEQ & MEAN/ & TIME OF FIRST & END & TIME SINCE & RESPI & TOTAL \\
\hline 6 & & NUM & TYPE & PULLSES & RESP(TICKS) & TIME(TICKS) & REIN. & MIN & RESPONSES \\
\hline 7 & $0-16$ & & DO & & & 56986 & 6 & 80.00 & 32 \\
\hline 8 & $0-40$ & 2 & DO & & & 56990 & 12 & 85.00 & 34 \\
\hline 9 & 0.4 & 3 & $\overline{D C}$ & 0 & 0 & 57371 & 18 & 85.00 & 34 \\
\hline 10 & $0-4$ & 4 & $D C$ & 0 & 0 & 57732 & 24 & 110.00 & 44 \\
\hline 11 & $0-4$ & 5 & $D C$ & 0 & 0 & 58093 & 30 & 107.50 & 43 \\
\hline 12 & $0-4$ & 6 & DC & 16 & 58253 & 58455 & 36 & 90.00 & 36 \\
\hline 13 & $0-4$ & 7 & $D C$ & 13 & 58478 & 58814 & 42 & 107.50 & 43 \\
\hline 14 & $0-4$ & 8 & $\overline{D C}$ & 3 & 58837 & 59176 & 48 & 162.50 & 65 \\
\hline 15 & $0-4$ & 9 & $\overline{D C}$ & 8 & 59356 & 59538 & 54 & 235.00 & 94 \\
\hline 16 & $0-4$ & 10 & DC & 14 & 59560 & 59897 & 60 & 210.00 & 84 \\
\hline 17 & $0-4$ & 11 & $D C$ & 26 & 59948 & 60259 & & & \\
\hline 18 & 0.4 & 12 & $D C$ & 26 & 60301 & 60619 & & & \\
\hline 19 & $0-4$ & 13 & $D E$ & 1 & & 60649 & & & \\
\hline 20 & & & & 0 & - & 60659 & & & \\
\hline 21 & $0-4$ & 14 & DI & 0.5 & 60669 & 60849 & & & \\
\hline$\overline{22}$ & $0-60$ & 15 & DO & & & 60853 & & & \\
\hline 23 & $0-4$ & 3 & $D C$ & 16 & 60974 & 61220 & & & \\
\hline 24 & $0-4$ & 4 & $D C$ & 17 & 61243 & 61581 & & & \\
\hline 25 & $0-4$ & 5 & $\overline{D C}$ & 17 & 61603 & 61943 & & & \\
\hline 26 & 0.4 & 6 & $D C$ & 14 & 61965 & 62303 & & & \\
\hline 27 & $0-4$ & 7 & $D C$ & 15 & 62326 & 62665 & & & \\
\hline 28 & $0-4$ & 8 & DC & 15 & 62688 & 63027 & & & \\
\hline 29 & $0-4$ & 9 & DC & 14 & 63049 & 63386 & & & \\
\hline 30 & 0.4 & 10 & $D C$ & 14 & 63418 & 63746 & & & \\
\hline 31 & $0-4$ & 11 & $\overline{D C}$ & 15 & 63769 & 64108 & & & \\
\hline 32 & $0-4$ & 12 & DC & 12 & 64155 & 64468 & & & \\
\hline $3 \sqrt{3}$ & 0.4 & 13 & $\mathrm{DE}$ & 1 & - & 64484 & & & \\
\hline 34 & & & & 0 & $\cdot$ & 64495 & & & \\
\hline 35 & $0-4$ & 14 & DI & 0.5 & 64505 & 64700 & & & \\
\hline 36 & $0-60$ & 15 & $D O$ & & & 64704 & & & \\
\hline 37 & $0-4$ & 3 & $D C$ & 0 & 0 & 65073 & & & \\
\hline 38 & 0.4 & 4 & $\mathrm{DC}$ & 0 & 0 & 65431 & & & \\
\hline 39 & $0-4$ & 5 & $\overline{D C}$ & 0 & 0 & 65792 & & & \\
\hline 40 & $0-4$ & & $\mathrm{DC}$ & 0 & 0 & 66154 & & & \\
\hline 41 & $0-4$ & 7 & $D C$ & 0 & 0 & 66516 & & & \\
\hline 42 & $0-4$ & 8 & $D C$ & 2 & 66709 & 66874 & & & \\
\hline 43 & $0-4$ & 9 & $D C$ & 7 & 66927 & 67235 & & & \\
\hline$\overline{44}$ & $0-4$ & 10 & $D C$ & 11 & 67268 & 67596 & & & \\
\hline 45 & $0-4$ & 11 & $\overline{D C}$ & 10 & 67618 & 67957 & & & \\
\hline 46 & $0-4$ & 12 & $D C$ & 9 & 68000 & 68319 & & & \\
\hline 47 & $0-4$ & 13 & DE & 1 & - & 68354 & & & \\
\hline
\end{tabular}

Figure 6. The data report generated by the fixed-interval 60 -sec schedule of reinforcement after the completion of three intervals and a summary analysis. The data report appears in columns $A$ through $I$, and the summary analysis appears in columns $J$ through $L$.

ple input, we designated this device as a high-speed counter by entering the letter $C$ in column $C$. The number 7 in column $\mathrm{D}$ commands the counter to detect the operation of a normally closed switch and to generate an output event when the ratio requirement is met. The output device, in this case a grain feeder, is specified by noting its byte number in column $\mathrm{E}$ and its bit number in column F. Column I specifies the ratio value, while column $J$ determines the duration of the output pulse. In this example we have specified a 4-sec feeder operation; the clock is set in 16-msec steps, so that an entered value of 250 results in a pulse duration of $4 \mathrm{sec}$. This is the largest value that can be specified in this manner. However, entering a 0 in this cell will result in the device being latched on indefinitely; a subsequent sequence could then turn the device off at any desired time. In column $K$, the letter $\mathrm{C}$ instructs the interface to sample Key 4 continuously, while in column $R$, the asterisk requests that a frequency count be kept. In column S, labeled "Criteria," note the number 40 . This indicates that the FR 5 schedule should execute 40 times before the program is ended in Sequences 4 and 5 . Sequences 4 and 5 turn off Keylight 40 and Houselight 16, respectively. The template is now complete and, if saved to disk in SYLK format, can be executed from Macontrol. This simple procedure was written for a single station. The real power of Macontrol, however, lies in its ability to extend this control to multiple stations easily.

The template shown in Figure 3 was generated by inserting blank rows into the FR 5 template and then add- 
HYPOTHETICAL DATA

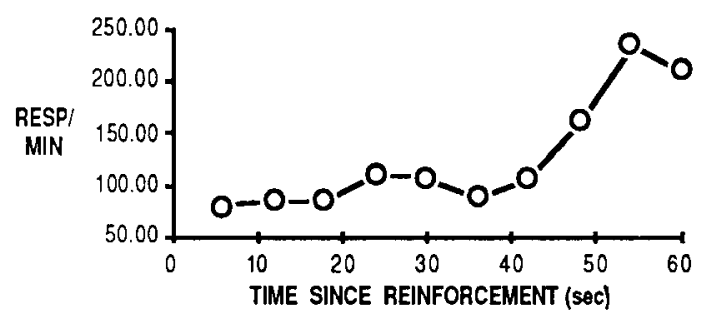

Figure 7. Response rates in successive tenths of the fixed interval. This graph was based on the summary analysis shown in Figure 6.

ing additional device numbers for each sequence. Note that there are now four rows associated with Sequence 1; these correspond to the houselights in four separate chambers. Similarly, the four rows associated with Sequence 3 sample the center kcy in each of the four boxes. The only other changes made were that we edited the bit numbers for the different grain feeders and changed the ratio value from 5 to 50 .

Another valuable feature of Macontrol is the data report. Figure 4 illustrates a portion of the report automatically generated by the FR 50 template. The report is organized by channel number, sequence number, and sequence type, and provides the number of pecks that occurred during each execution of a sequence, the time of the first response in that sequence, and the time at which each sequence was completed. All of the times are indicated in units of $1 / 60 \mathrm{sec}$ since system startup. Using Excel, it is easy to extract a distribution of pauses, as well as the local response rate for each ratio in the session. The format of the data report, however, depends on the way the control template is writ- ten, and reports may contain much more detailed data. Figure 5 shows a template to collect frequency data for each tenth of a fixed-interval 60 -sec schedule.

The data report generated by this template is shown in Figure 6. Data for three intervals in the session are visible; column D of rows 12-21 contains bin response data for the first interval. The next column shows time of the first response for each bin. A time of 0 is recorded when no responses actually occur in a bin. To summarize response rates for the session, the user may use Excel's "paste-with-sum" option to sum the total responses occurring in each tenth of the fixed interval (FI). The summary data shown in column $\mathrm{L}$ were obtained in this manner. A simple formula converts the frequencies to rates, and the information in columns J and $\mathrm{K}$ can be "selected" with the mouse and "pasted" into a chart. The resulting chart of these data is shown in Figure 7.

\section{CUSTOM SOFTWARE AND DATA ANALYSIS}

While Macontrol can accomplish much, including variable schedules, multiple schedules, and certain concurrent schedules, the package has limitations. In general, the more complex the procedure, the less desirable it is to use Macontrol-generated programs. For these cases, and when the Macintosh serves as a stand-alone research station for human operant research, we write routines using ZBasic. In all cases, however, data are written to disk as tab-delimited text files that can be opened by Excel for further analysis.

\section{Data File Formats}

At the conclusion of each session, our customized software stores an itemized data file that records session

\begin{tabular}{|c|c|c|c|c|c|}
\hline & A & 8 & $\mathrm{c}$ & 0 & $\mathbf{E}$ \\
\hline 1 & TIME & EVENIS & SCHEDULE & \multicolumn{2}{|c|}{ Description of code } \\
\hline 2 & 162645 & 161 & & \multicolumn{2}{|c|}{ Houselight onset } \\
\hline 3 & 162816 & 201 & & \multicolumn{2}{|c|}{ Left red key light on } \\
\hline 4 & 162974 & 521 & 0 & \multicolumn{2}{|c|}{ Right amber key light on } \\
\hline 5 & 163010 & 1 & & Left key peck & \\
\hline 6 & 163030 & 1 & & Left key peck & \\
\hline 7 & 163050 & 1 & & Left key peck & \\
\hline 8 & 163070 & 2 & 0 & Right key peck & \\
\hline 9 & 163090 & 200 & & \multicolumn{2}{|c|}{ Left red key light off } \\
\hline 10 & 163110 & 241 & & \multicolumn{2}{|c|}{ Left green key light on } \\
\hline 11 & 163130 & 1 & 2 & Left key peck & \\
\hline 12 & 163150 & 1 & & Left key peck & \\
\hline$\sqrt{3}$ & 163170 & 1 & 2 & Left key peck & \\
\hline 14 & 163192 & 601 & & \multicolumn{2}{|c|}{ Feeder operated } \\
\hline 15 & 163248 & 240 & 2 & & \\
\hline 16 & 163302 & 520 & 2 & & \\
\hline 17 & 163432 & 600 & 2 & & \\
\hline 18 & 163450 & 1 & 2 & & \\
\hline 19 & 163476 & 1 & 2 & & \\
\hline 20 & 163502 & 2 & 0 & & \\
\hline
\end{tabular}

Figure 8. A segment of an itemized data file produced by our custom software. 


\begin{tabular}{|r|r|r|r|r|r|r|r|r|}
\hline & A & B & \multicolumn{1}{c|}{ C } & O & E & \multicolumn{1}{c|}{ F } & G & H \\
\hline 1 & Raw Data & & & & & Analyses & & \\
\hline 2 & BIRD 1 & VI $60^{*}$ VI $60^{\circ}$ & & & & RESP/MIN & & \\
\hline 3 & DATE & RESP RED & RESP GRN & TIME RED & TIME GRN & RED & GRN & RED/GRN \\
\hline 4 & $5 / 18 / 88$ & 2500 & 3100 & 1700 & 1900 & 41.67 & 51.67 & 0.81 \\
\hline 5 & $5 / 19 / 88$ & 2900 & 3075 & 1755 & 1845 & 48.33 & 51.25 & 0.94 \\
\hline 6 & $5 / 20 / 88$ & 2600 & 3000 & 1745 & 1855 & 43.33 & 50.00 & 0.87 \\
\hline 7 & $5 / 21 / 88$ & 2750 & 3100 & 1780 & 1820 & 45.83 & 51.67 & 0.89 \\
\hline 8 & $5 / 22 / 88$ & 2950 & 3050 & 1805 & 1795 & 49.17 & 50.83 & 0.97 \\
\hline 9 & $5 / 23 / 88$ & 3050 & 3075 & 1810 & 1790 & 50.83 & 51.25 & 0.99 \\
\hline 10 & $5 / 24 / 88$ & 3125 & 3050 & 1805 & 1795 & 52.08 & 50.83 & 1.02 \\
\hline 11 & $5 / 25 / 88$ & 3200 & 3025 & 1820 & 1780 & 53.33 & 50.42 & 1.06 \\
\hline 12 & $5 / 26 / 88$ & 3250 & 2950 & 1845 & 1755 & 54.17 & 49.17 & 1.10 \\
\hline 13 & $5 / 27 / 88$ & 3350 & 3000 & 1850 & 1750 & 55.83 & 50.00 & 1.12 \\
\hline 14 & & & & & & & & \\
\hline
\end{tabular}

Figure 9. A condition summary file based on the hypothetical concurrent schedules experiment.

events. The format of these files varies, depending on the application used to produce the file. Whenever possible, such as when the Macintosh controls only one station, the itemized data files date and code each session event.

Consider a hypothetical experiment in which a pigeon may peck either of two keys on a concurrent schedule of reinforcement, where the left key is the schedule key and the right key is a changeover key. Figure 8 shows a segment of an itemized data file that would be produced by such a procedure. The first column dates each session event in "ticks," units of $1 / 60 \mathrm{sec}$ since the onset of the system. The second column identifies the event with an event code. The numbers 1 and 2 refer to pecks on the left and right keys, respectively, while the three-digit numbers indicate the onset or offset of a stimulus event. The first two digits of the stimulus-event codes give the channel number for the stimulus-changing states, while the third digit indicates whether the change is a stimulus onset or offset. For example, the numbers 201 and 200 refer to the onset and offset, respectively, of the left, red keylight. The third column identifies the current schedule. Our students use Excel's database functions to extract information from these files, such as the number of responses made during the green light.

These data may be further analyzed by using an analysis template pasted into a condition summary file. Figure 9 shows a condition summary file for the hypothetical experiment described above. Formulas were pasted into the right side of the worksheet and copied down the page. When the raw data is pasted into the appropriate location of the worksheet, the calculated measures appear in the formula cells. This file summarizes the results of a given condition, and it may be used to produce a graph of daily responding in the manner described above.

\section{SUMMARY}

All of the operations discussed, from opening Macontrol to displaying the final chart, can be automated with macros. Our undergraduates are therefore able very quickly to generate experiment-control codes, run the session, summarize the day's data, and view it graphically. To perform additional calculations, students can create a template worksheet that contains the formulas for the desired measures. Then they can electronically copy and paste the data into the correct location in the template worksheet, and the computed measures will appear in the cells containing the formulas.

The combined uses of Macontrol, custom applications, and the Excel worksheet enable us to support the apprenticeship program with a system that is easy to learn and use, and that handles data electronically, from initial data collection through the final preparation of graphs and reports. We have been very encouraged by the initial accomplishments of our undergraduates.

\section{REFERENCES}

Chute, D. L., Gaeman, D., \& Ziegler, T. (1987). MacLaboratory Controller: A switch and A/D interface between Apple's Macintosh and peripheral apparatus. Behavior Research Methods, Instruments, \& Computers, 19, 205-209.

Cooper, L. D., Garcia, R., \& Gibbon, J. (1988). The labtop Macintosh: An interface and communications software for experiment control of animal learning research. Behavior Research Methods, Instruments, \& Computers, 20, 88-92.

Weisman, R., \& Palya, W. L. (1988). Development and operating environments for a network of Walter/Palya experiment controllers on the Macintosh computer. Behavior Research Methods, Instruments, \& Computers. 20, 93-96. 